\title{
Lymphoma transformation of tumor infiltrating lymphocytes observed in testicular patient-derived xenograft models
}

\author{
K. KALAVSKA ${ }^{1-3}$, L. KUCEROVA ${ }^{3}$, S. SCHMIDTOVA ${ }^{3}$, L. TORO ${ }^{3}$, Z. KOZOVSKA ${ }^{3}$, L. PLANK $^{4}$,

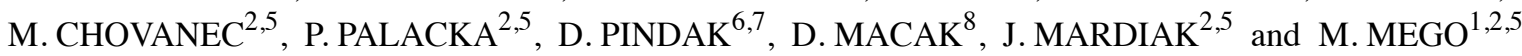 \\ ${ }^{1}$ Translational Research Unit, ${ }^{2}$ Second Department of Oncology, Faculty of Medicine, Comenius University \\ and National Cancer Institute, 83310 Bratislava; ${ }^{3}$ Laboratory of Molecular Oncology, Cancer Research Institute, \\ Biomedical Research Center of The Slovak Academy of Sciences, 84505 Bratislava; ${ }^{4}$ Department of Pathological Anatomy, \\ Jessenius Faculty of Medicine, Comenius University and University Hospital, 03601 Martin; ${ }^{5}$ Department of Oncology, \\ National Cancer Institute, 83310 Bratislava; ${ }^{6}$ Department of Surgery, Slovak Medical University, 83303 Bratislava; \\ Departments of ${ }^{7}$ Surgical Oncology and ${ }^{8}$ Pathology, National Cancer Institute, 83310 Bratislava, Slovakia
}

Received May 1, 2018; Accepted September 19, 2018

DOI: 10.3892/or.2018.6769

\begin{abstract}
Testicular germ cell tumors (TGCTs) are highly sensitive to cisplatin-based chemotherapy. Nevertheless, there are metastatic tumors that do not completely respond to front-line chemotherapy. For these tumors, surgical resection of residual masses is necessary to achieve long-term disease control. Resected tissues represent valuable clinical material, which may be used for the engraftment into immunocompromised mice to produce patient-derived xenografts (PDXs). They typically maintain similarities to the parental tumors and therefore serve as more realistic preclinical models. Moreover, a correlation between PDX treatment outcomes and clinical response to chemotherapy has been previously described. The aim of the present study was to establish and characterize TGCT patient-derived xenografts. These originated from retroperitoneal lymph node metastases infiltrated with TGCTs following previous cisplatin-based chemotherapy, in order to analyze novel treatment options for cisplatin-resistant testicular tumors. We generated two testicular patient-derived xenograft models in SCID beige male mice. Immunohistochemical analyses demonstrated that histological characteristics of the primary tumor were not retained, and transformation into lymphoma, and eventually plasmocytoma, was observed. A potential explanation for the lymphoma transformation observed in PDXs may include tumor-infiltrating lymphocytes (TILs) in xenografted
\end{abstract}

Correspondence to: Professor M. Mego, Translational Research Unit, Faculty of Medicine, Comenius University and National Cancer Institute, Klenova 1, 83310 Bratislava, Slovakia

E-mail: misomego@gmail.com

Key words: patient-derived xenograft model, testicular germ cell tumors, resistance to chemotherapy, retroperitoneal lymph node dissection, lymphoma transformation, tumor infiltrating lymphocytes samples of patients, which are transformed following engraftment into immunodeficient recipient mice. Based on these data, we indicated that lymphomagenesis prevention and terminal differentiation represent new challenges in the establishment of PDX models derived from patients with germ cell tumors.

\section{Introduction}

Testicular germ cell tumors (TGCTs) represent the most common type of solid malignancy among young men between the ages of 20 and 40 (1). TGCTs are highly curable malignancies, with current cure rates exceeding $70-80 \%$, even in patients with disseminated disease. However, there are patients with metastatic testicular cancer who do not achieve a durable complete remission with cisplatin-based chemotherapy. Surgical resection of residual masses is necessary to achieve long-term disease control in these patients. The presence of teratoma components in these residual masses has important clinical consequences. Teratoma in the retroperitoneum may grow, which is a structure characterized by enlarging metastatic masses without marker elevation, termed growing teratoma syndrome. Alternatively, patients may develop high-grade malignant components, including sarcomas and primitive neuroectodermal tumors $(2,3)$. In addition, only $20-25 \%$ of the patients progressing/relapsing after first-line chemotherapy can be cured by standard dose or high-dose chemotherapy with autologous hematopoietic stem-cell rescue (4-6). Patients who fail to achieve remission following conventional therapy have an extremely poor prognosis and the vast majority of them will eventually die of the disease (7-9). Therefore, a better understanding of resistance to chemotherapy together with developing novel therapeutic strategies based on the molecular and genetic characteristics of the tumor is required (7).

In order to achieve an effective prediction of clinical efficacy of new approaches in cancer drug development, more realistic models reflecting the heterogeneity of patients are required. Established cancer cell lines and monocellular layers 
of tumor cells cultivated in vitro, as well as mouse xenografts derived from those tumor cells have been used as the standard toolkit in preclinical drug evaluation and biomarker identification. However, despite several advantages, recent data suggests that the cells grown in culture and xenografts derived from these expanded cells adapted to growth in artificial culture conditions have principally diverged from the primary tumors from which they were derived (10-12).

Patient-derived xenografts (PDXs) are tumor models based on transplanting surgically resected tumor tissue samples from donors directly into immunodeficient mice. In comparison with cell line culture, PDXs maintain more similarities to the parental tumors and therefore serve as a more realistic preclinical model with strong predictive power in the translation of new anti-cancer agents to clinical practice $(13,14)$. PDXs retain histology as well as the gene expression profiles of their donor tumors $(15,16)$. The correlation between PDX tumors and clinical response to chemotherapy has also been described (17). In order to investigate treatment failure in patients with TGCT, there has been increasing interest in the establishment of PDX models that realistically model the disease and have the potential to predict clinical outcomes for novel therapeutic strategies (18). Several research groups have attempted to establish PDX models derived from testicular cancer. Abraham et al xenografted human non-seminomatous germ cell tumors into SCID mice (19). The study carried out by Castillo-Avila et al established orthotopic models for cisplatin sensitive non-seminomas, as well as for cisplatin-resistant choriocarcinoma variants induced in mice. Notably, only non-seminomatous tumors (predominantly choriocarcinomas, embryonal carcinoma, yolk sac tumor or mixed TGCTs) were successfully implanted in mice. No pure seminomas that presented as primary tumors have been grown as xenografts (20). To the best of our knowledge, only the study by de Vries et al established a PDX model from a metastatic lesion of a patient with refractory testicular cancer (21). The engraftment of tissue samples obtained from the metastatic lesions of patients with progressive disease after standard cisplatin-based chemotherapy treatment represents an ideal model system for studying mechanisms implicated in resistance to cisplatin, as well as in clarification of the mechanisms involved in growing teratoma syndrome.

In the present study, we aimed to establish and characterize testicular cancer patient-derived xenografts, from retroperitoneal lymph nodes infiltrated with TGCTs after cisplatin-based chemotherapy administration. We present our data demonstrating that two successfully implanted patient samples diverged from the original tumor histology and in vivo passage resulted in terminal differentiation into lymphoma and plasmocytoma. Consequently, these samples could not be serially propagated in vivo.

\section{Materials and methods}

Study patients. As a part of an ongoing translational study (protocol IZLO1; chair, M. Mego) TGCT patients who underwent retroperitoneal lymph node dissection between April 2015 and August 2015 at the National Cancer Institute of Slovakia were enrolled. Patients with concurrent malignancies other than non-melanoma skin cancer in the previous 5 years were excluded from the study. Data regarding age, tumor histological subtype, clinical stage, histological subtype and number of metastatic lesions, previous chemotherapy were recorded for all patients. The Institutional Review Board of the National Cancer Institute (Bratislava, Slovakia) approved the present study and all patients provided written informed consent.

Tissue sampling. Clinical samples for the PDX tumor model were obtained immediately after resection and transferred into RPMI-1640 culture medium on wet ice for engraftment within $24 \mathrm{~h}$. Tumor tissue collected from each patient was separated into three sections under sterile conditions. One section was cryopreserved in liquid nitrogen and stored at $-80^{\circ} \mathrm{C}$; the second was fixed in $10 \%$ neutral buffered formalin solution and embedded in paraffin for histopathological analysis of the implanted tumor; and the third portion of the tumor (representing F0 generation) was used for engraftment in SCID beige male mice. Baseline characteristics of donor patients are provided in Table I.

Establishment of the patient tumor-derived xenografts. Fresh tumor tissue was mechanically dissociated into small pieces in $0.5 \mathrm{ml}$ RPMI-1640 cultivation media. Suspension was collected into a sterile syringe and passed through an $18 \mathrm{G}$ needle three times. The final volume was determined and an equivalent volume of ECM (ECM Gel from Engelbreth-Holm-Swarm murine sarcoma; cat. no. 1270; Sigma-Aldrich; Merck KGaA, Darmstadt, Germany) was added on ice.

Six to eight weeks old SCID beige male mice (CD17 Cg-Prkdscid Lystbg/Crl) were bilaterally subcutaneously injected with 150-200 $\mu \mathrm{l}$ suspension. The mice were housed under controlled environmental conditions $\left(24 \pm 2^{\circ} \mathrm{C}\right.$, relative humidity $40-60 \%$ and a 12-h light/dark cycle). Animals had free access to food and water ad libitum and were regularly monitored for their weight, the presence of any pathological conditions and tumor growth. Tumor volume was determined based on the caliper measurements of two perpendicular diameters and calculated according to the following formula: Volume $=$ length $\mathrm{x}$ width $/ 2$. Animals were sacrificed when any of the tumor diameters exceeded $10 \mathrm{~mm}$ or tumor xenografts exhibited signs of necrosis and bleeding. Tumor xenografts were collected for further analysis. Each tumor was divided into two: One section was fixed in buffered formalin and embedded in paraffin for histological and immunohistochemical analyses and the other was submerged in PBS for preparation of the suspension as described above. An aliquot from the suspension was used for DNA isolation.

Animal experiments were performed in the approved animal facility (license number SK PC 14011), as approved by the Institutional Ethics Committee and by the national competence authority (State Veterinary and Food Administration of the Slovak Republic; registration no. Ro 3108/14-221). Experiments were performed in compliance with the Directive 2010/63/EU of the European Parliament and the European Council and the Regulation 377/2012 on the protection of animals used for scientific purposes.

Histological and immunohistochemical evaluation of PDX tumors. Histological examinations were based on paraffin 
sections using H\&E, Giemsa and PAS staining and Gomori's silver impregnation staining method to determine the growth pattern and tumor cell histopathology.

For determination of the tumor phenotype, acomplex panel approach was used. This initially involved the detection of i) lymphoid B-cell markers (PAX5, CD20, CD138, kappa and lambda Ig light chains) and T-cell markers (CD3, CD5), as well as ii) placental alkaline phosphatase (PLAP) and cytokeratins (using AE1/AE3, CK7 and CK20 antibodies) in both further documented PDX tumors. Following this, more detailed phenotypic analysis to contribute to the diagnosis of both cases was performed, including evaluation of the expression of antigens Bcl-2, BCL6, CD10, MUM1/IRF-4, CD30, CD15, CD56, as well as latent membrane protein (LMP) Epstein-Barr virus. Deparaffinization, rehydration and target retrieval with the Target Retrieval Solution High pH (pH 9.0; Dako; Agilent Technologies, Inc., Santa Clara, CA, USA) or EDTA (pH 9.0) at $96^{\circ} \mathrm{C}$ was performed in the PT Link (Dako PT100; Dako; Agilent Technologies, Inc.). Slides were subsequently processed on the Autostainer Link 48 (Dako AS480; Dako; Agilent Technologies, Inc.) using an automated staining protocol validated for the individual antibodies, or mechanically using kit EnVision FLEX (Agilent Technologies, Inc.), High pH (Link) in agreement with the manufacturer's recommendations. Reagents utilized in addition to the mentioned components included a FLEX antibody diluent, FLEX wash buffer and a hematoxylin counterstain (Dako; Agilent Technologies, Inc.). IHC-stained slides were mounted in non-aqueous, permanent mounting media.

DNA extraction and qPCR analysis. The ISOLATE II FFPE RNA/DNA kit (Bioline, London, UK) was used for the isolation and purification of genomic DNA from formalin-fixed paraffin-embedded tissue samples. We proceeded according to the manufacturer's instructions. The isolated genomic DNA was analyzed by qPCR. Reactions were performed using a Maxima probe qPCR Master Mix (Fermentas; Thermo Fisher Scientific, Inc., Waltham, MA, USA). qPCR was carried out with a CFX96 thermocycler (Bio-Rad Laboratories, Inc., Hercules, CA, USA) according to the following thermocycling parameters: Pre-treatment at $50^{\circ} \mathrm{C} 2 \mathrm{~min}$, initial denaturation at $95^{\circ} \mathrm{C}$ for $10 \mathrm{~min}$, followed by 50 cycles at $95^{\circ} \mathrm{C}$ for $20 \mathrm{sec}$ and $60^{\circ} \mathrm{C}$ for $1 \mathrm{~min}$. The plate was then read. Final annealing was peformed at $60^{\circ} \mathrm{C}$ for $10 \mathrm{~min}$ followed by a cooling step to $7^{\circ} \mathrm{C}$. The specific primers and probes used in duplex qPCR are described in Table II. The mouse Rapsyn (receptor-associated protein at the synapse) gene was used as an internal control. The quantity of the PCR product was calculated by the $\mathrm{Cq}$ value (22). The positive control DNA was isolated from human breast cancer cells MDA-MB-231 (ATCC ${ }^{\circledR}$ HTB-26 ${ }^{\mathrm{TM}}$; ATCC, Manassas, VA, USA). EGFP-expressing MDA-MB-231 were kindly provided by Dr Miroslava Matuskova (23). DNA isolated from the mouse cell line NIH/3T3 (ATCC ${ }^{\circledR}$ CRL-1658 ${ }^{\mathrm{TM}}$; ATCC) was used as a negative control. Evaluation of human specificity of $\beta$-globin amplification was demonstrated using a 2-fold dilution for 100 to 0 ng human gDNA per assay in PCR grade water, and in 0 to 100 ng murine gDNA per assay. The quantity of human gDNA was calculated according to the calibration curve prepared as stated above. The amount of human $\beta$-globin was related to $150 \mathrm{ng}$ total DNA. 
Table II. Sequences of primers used in this study.

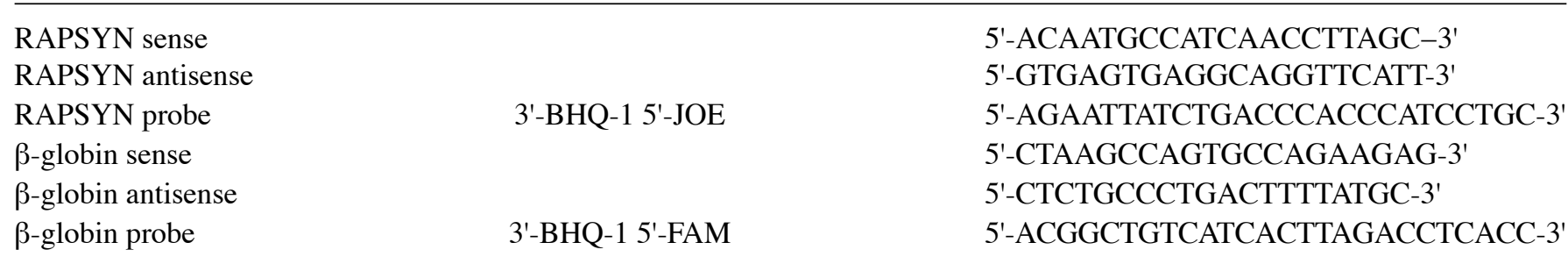

A

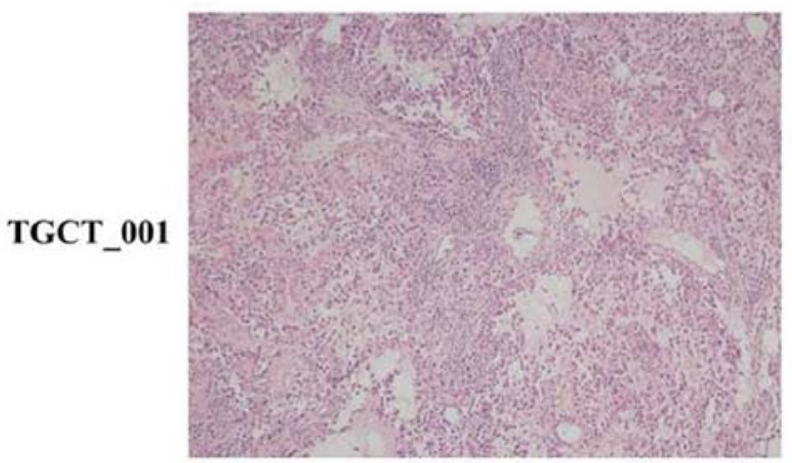

B

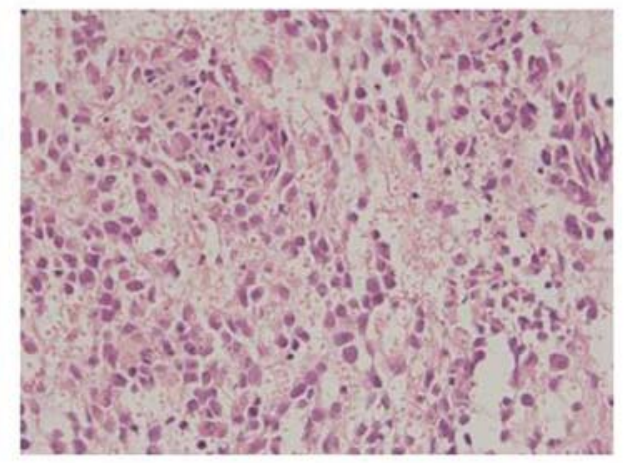

Figure 1. Representative histology of primary patient's tumor sample no. TGCT_001. (A) Tumor tissue slides stained with hematoxylin and eosin (magnification, $\mathrm{x} 10$ ). (B) Tumor tissue slides stained with hematoxylin and eosin (magnification, $\mathrm{x} 40$ ).

A

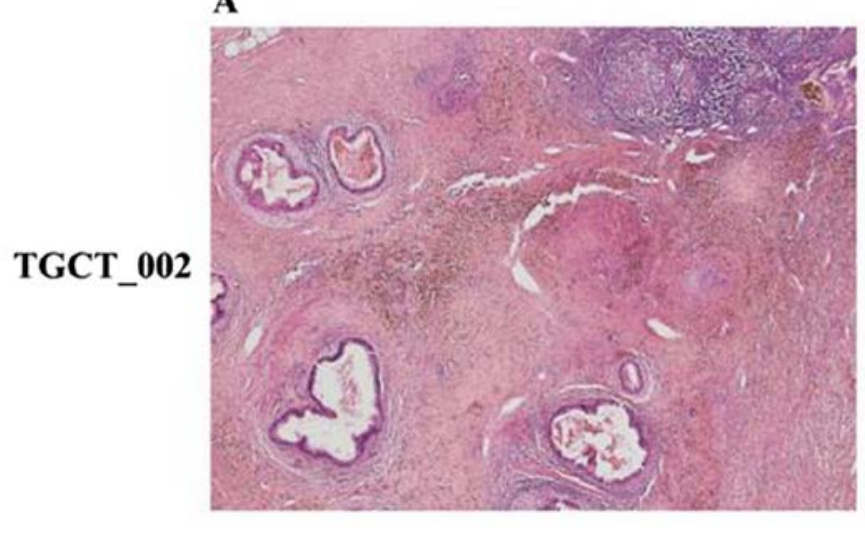

B

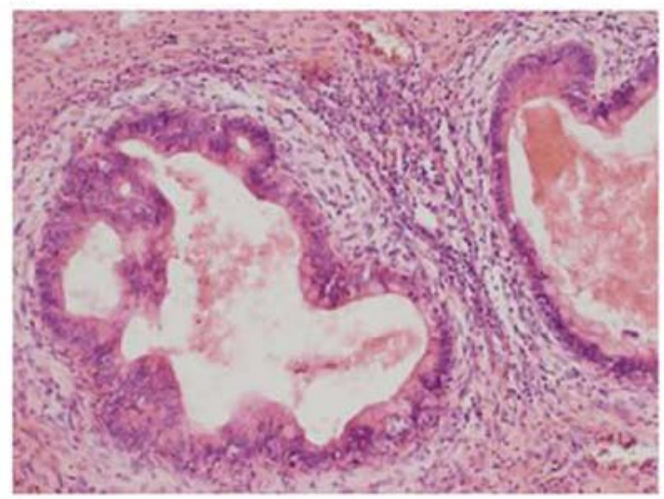

Figure 2. Representative histology of primary patient's tumor specimen no. TGCT_002. (A) Tumor tissue slides stained with hematoxylin and eosin (magnification, x10). (B) Tumor tissue slides stained with hematoxylin and eosin (magnification, $\mathrm{x} 40$ ).

\section{Results}

Baseline characteristics. Tumorspecimens from retroperitoneal lymph nodes infiltrated with TGCTs from three testicular cancer patients were engrafted into SCID mice in order to establish a PDX model. The summary of donor patient characteristics is presented in Table I. The median age of patients enrolled in this study was 45 years (range, 30-46 years). Histology of the primary tumors determined the following subtypes: Seminomas (cases TGCT_001 and TGCT_003) and non-seminoma with embryonal carcinoma, yolk sac tumor, immature teratoma and seminoma components (case TGCT_002). All patients were pretreated with cisplatin-based chemotherapy. In one patient, a retroperitoneal lymph node biopsy was performed prior to starting second line chemotherapy (case TGCT_001) to histologically confirm disease recurrence, while other patients underwent retroperitoneal lymph node dissection after finishing first line chemotherapy (case TGCT_002) and salvage chemotherapy (case TGCT_003). The histology of post chemotherapy retroperitoneal lymph node dissection specimens from cases TGCT_001 and TGCT_003 representing F0 generation determined that the histological subtype was correlated with primary tumor histology (immunohistochemical positivity for PLAP and CD117 and negative for CD30), while in case TGCT_002, RPLN metastasis was derived only from a teratocarcinoma component (immunohistochemical positivity for CK20, CEA, CDX2 and negative for CK7) (Figs. 1 and 2). 
A

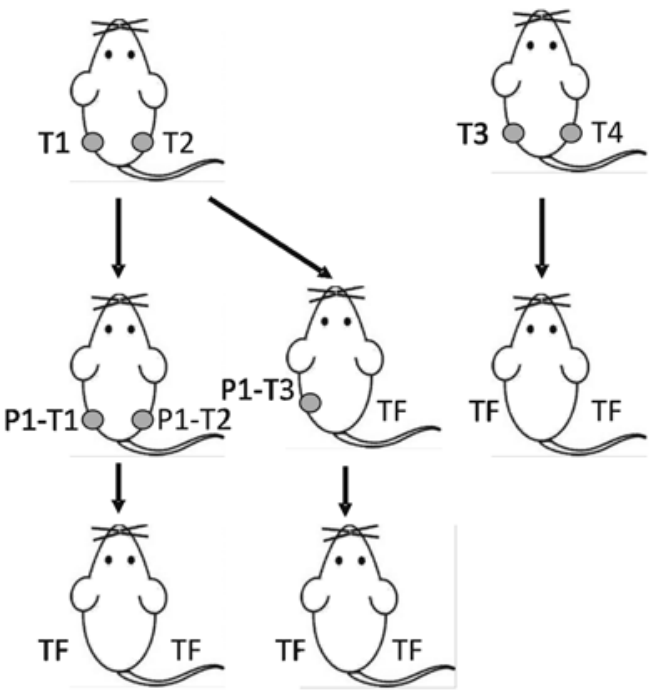

Patient-derived xenografts $4 / 4$ tumors growing

Patient-derived xenografts $1^{\text {st }}$ in vivo passage $3 / 6$ tumors growing

Patient derived xenografts $2^{\text {nd }}$ in vivo passage

$0 / 4$ tumors growing
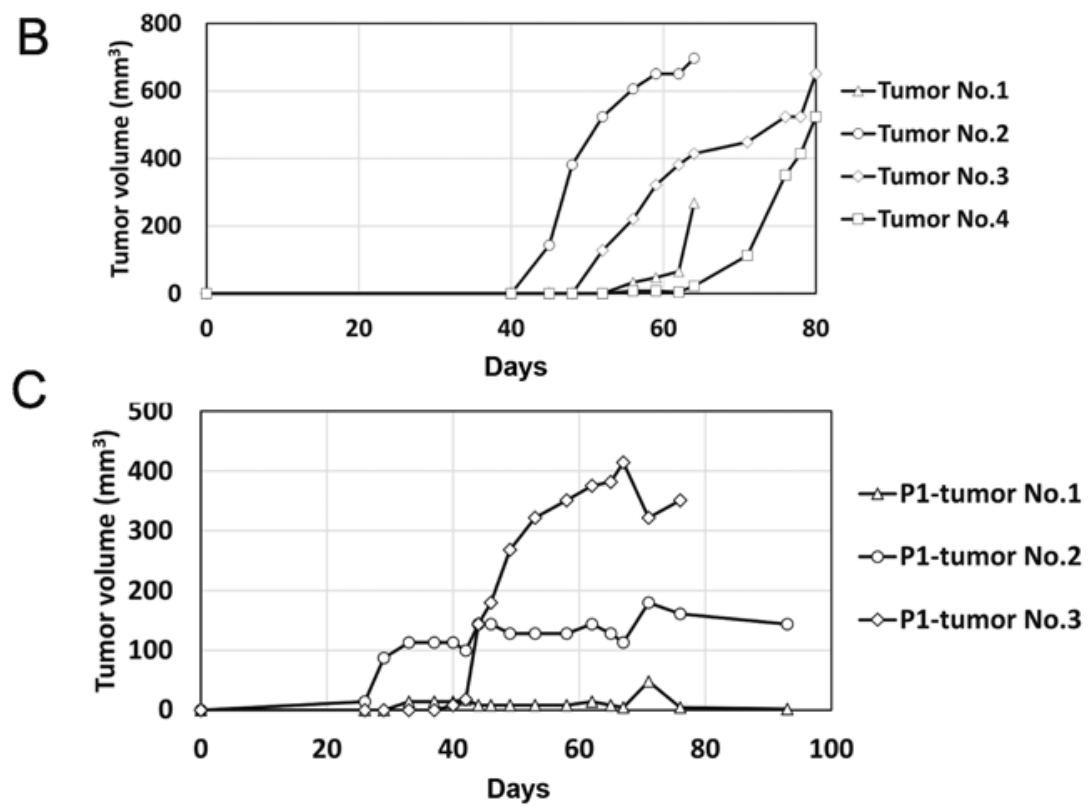

Figure 3. Establishment of the PDX model from the tumor sample no. TGCT_001. (A) Schematic overview of mouse inoculation. (B) Growth of primary tumor xenografts on immunodeficient mice. (C) Growth of tumor xenografts derived from the primary tumor xenograft upon passage in vivo.

Establishment of PDX models. To establish PDX models from fresh tumor tissue samples, they were mixed with ECM gel and subcutaneously engrafted into F1 mice. A total of 3 retroperitoneal lymph node metastases tissue sample were xenografted. Since these tissue samples were residual tumors in patients who underwent cisplatin-based chemotherapy, we supposed that they also contained tumor cells resistant to cisplatin. The baseline histological and clinical characteristics of the successfully implanted tissue samples of patients are summarized in Table I. In all cases, the post-chemotherapy treatment tissue samples representing F0 generation were grafted. Growing xenografts were established from the two out of the three inoculated patient samples. The first sample, designated TGCT_001, originated from a retroperitoneal metastatic seminoma and produced four tumor xenografts. These were subsequently injected into F2 mice as schematically depicted in Fig. 3A. The first two tumors were dissociated and injected
64 days after the experiment start date, and produced 3 tumors out of 4 inoculates. The other two tumors were dissociated and injected 80 days post-implantation, but did not produce any tumors. In contrast to the first injection, when exponential growth was observed in all four tumors after 40-55 days post-injection, the second injection produced only one exponentially growing xenograft (Fig. $3 \mathrm{~B}$ and $\mathrm{C}$ ). The other two injections produced palpable persistent tumor masses of stable volume. Subsequent inoculation of the suspension derived from these three xenografts did not produce any tumors in the next in vivo passage.

The second sample, designated TGCT_002, originated from a retroperitoneal metastatic mixed germ-cell tumor with embryonal carcinoma, yolk sac, teratoma and seminoma components. This sample produced three tumor xenografts. Exponentially growing tumorsweresubsequently injected into F2 mice 56 days after the experiment start date, as 

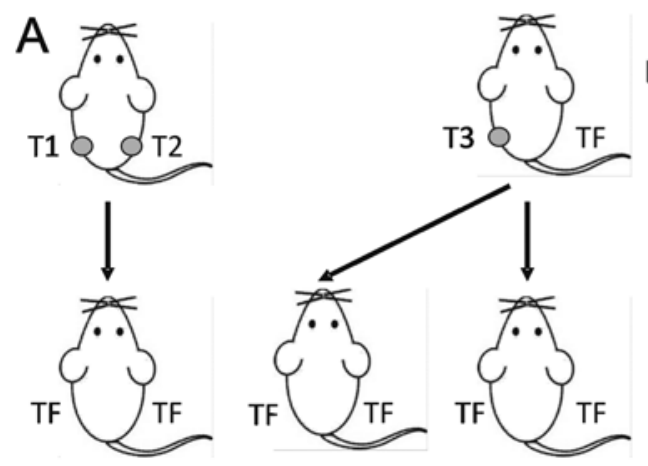

Patient-derived xenografts $3 / 4$ tumors growing

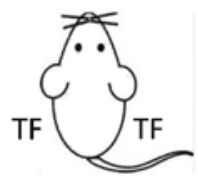

Patient-derived xenografts

$1^{\text {st }}$ in vivo passage

$0 / 6$ tumors growing

\section{B}

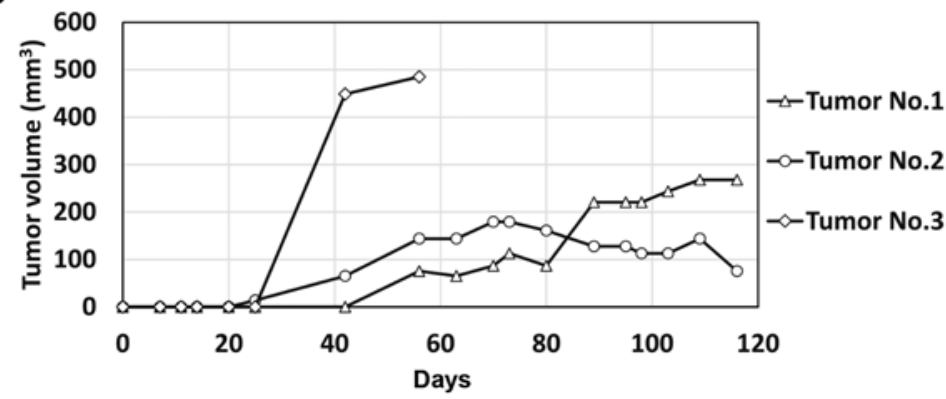

Figure 4. Establishment of the PDX model from the tumor sample no. TGCT_002. (A) Schematic overview of mouse inoculation. (B) Growth of primary tumor xenografts on immunodeficient mice.

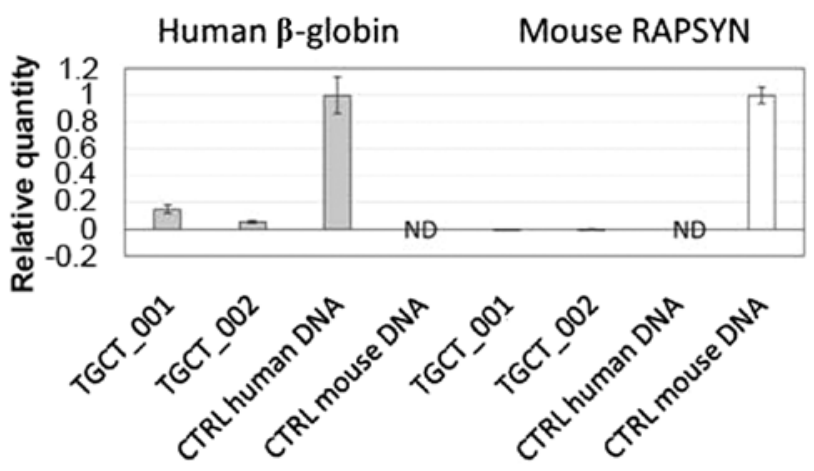

Figure 5. qPCR analysis of human $\beta$-globin and mouse Rapsyn in successfully engrafted samples TGCT_001 and TGCT_002. The quantity of PCR products was calculated by $\mathrm{Cq}$ value. The positive control DNA was isolated from human breast cancer cells MDA-MB-231/EGFP (ATCC ${ }^{\circledR}$ HTB-26 $^{\mathrm{TM}}$ ). DNA isolated from the mouse cell line NIH/3T3 (ATCC ${ }^{\circledR}$ CRL-1658 ${ }^{\mathrm{TM}}$ ) was used as a negative control. Evaluation of human specificity of $\beta$-globin amplification was demonstrated using a 2-fold dilution for 100 to 0 ng human gDNA per assay in PCR grade water and in 0 to $100 \mathrm{ng}$ murine gDNA per assay.

schematically depicted in Fig. 4A. The two other tumors were dissociated and injected 116 days post-implantation, but none of the inoculations produced any tumor. We observed exponential growth 25 days post-injection in this case, in one tumor only (Fig. 4B). The other two injections produced palpable persistent tumor masses that changed volume at an extremely slow rate. Subsequent inoculation of the suspension derived from these three xenografts did not produce any tumors in the next in vivo passage.

Molecular analysis confirmed the presence of human specific $\beta$-globin sequences in both successfully engrafted samples (Fig. 5).
Histological and immunohistochemical analysis of PDX tumors. Histological and immunohistochemical analysis of established PDX tumors demonstrated that the original histology was not maintained in any of the cases. Both successfully engrafted tumor tissues obtained from F1 mice displayed a lymphocytic dominant pattern, regardless of the tumor histology of patients (seminoma vs. mixed germ cell tumor). These cases were analyzed to determine their detailed histological and immunohistochemical features.

The histological analysis of case no. TGCT_001 exhibited diffuse proliferation of prevailing medium-sized lymphoid mononuclear blasts of centroblastic and immunoblastic morphology, with few intermingled and dispersed multinucleated giant blasts resembling Sternberg-Reed cells. Irrespective of the tumor cell size, all cells expressed immunohistochemical positivity for PAX5, CD20, CD30 (positivity appeared in $>70 \%$ of the mononuclear blasts and multinucleated giant cells), Bcl-2 and MUM1/IRF-4 together with a high proliferation rate (Ki-67 index, $70 \%$ ). By contrast, they were negative for CD3, CD5, CD15, BCL6, CD10, CD138, PLAP and cytokeratins. Twenty to thirty percent of tumor cells co-expressed p53 protein and LMP, and $\sim 40 \%$ of tumor cells also had Myc protein nuclear positivity (Fig. 6). According to all these results, we may conclude that PDX tumor no. TGCT_001 represented an anaplastic variant of a CD $20^{+} / \mathrm{CD} 30^{+}$diffuse large B-cell lymphoma.

The case no. TGCT_002 was characterized by diffuse infiltration of uniform cells with the morphology of mature, predominantly mononuclear plasma cells of Marschalko-type. These cells presented with an immunohistochemical phenotype of clonal plasmacytic proliferation with cytoplasmic Ig of 1 light chain type, were positive for CD138 and MUM1/IRF-4, and negative for PAX5, CD20, 
A

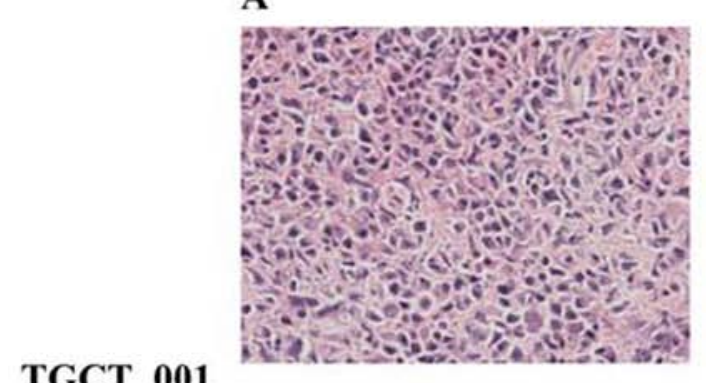

TGCT_001

D

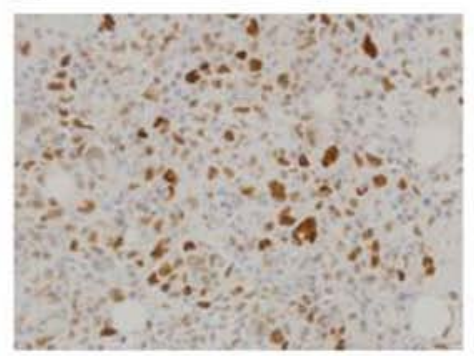

B

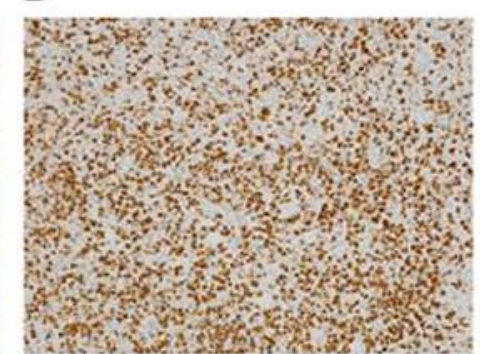

E

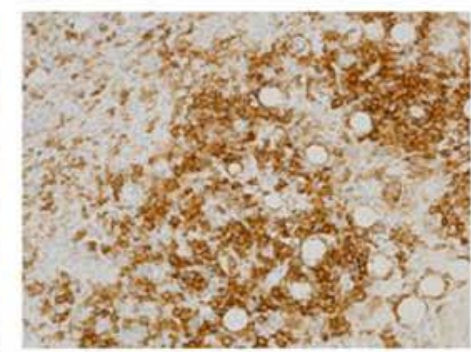

C

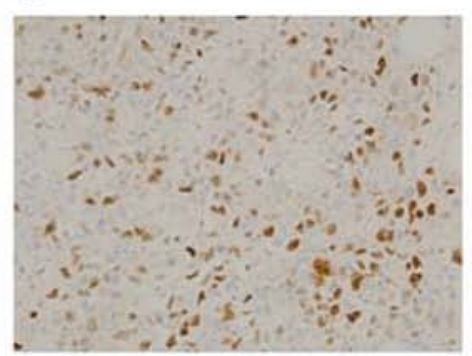

F

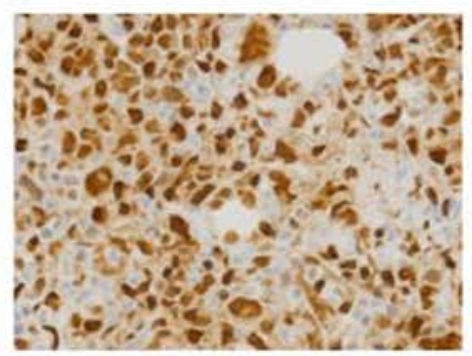

Figure 6. Representative histology of the PDX from seminoma tumor sample no. TGCT_001, exhibiting signs of diffuse large B cells lymphoma. (A) Tumor tissue slides stained with hematoxylin and eosin (magnification, x60). (B) Tumor tissue slides stained with proliferation marker Ki-67 antibody (magnification, x20). (C) Immunohistochemical staining of c-Mycin sections obtained from tumor tissue sample TGCT_001 (magnification, x40). (D) Immunohistochemical examination of p53 in analyzed tumor tissue slides (magnification, x40). (E) Positive-CD30 staining (magnification, x20). (F) Tumor tissue slides stained with PAX5 antibody (magnification, x60).

A

TGCT_002

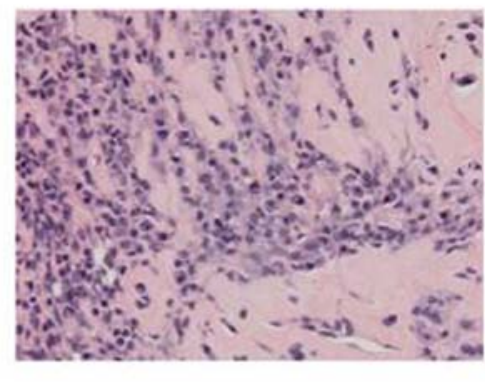

\section{C}

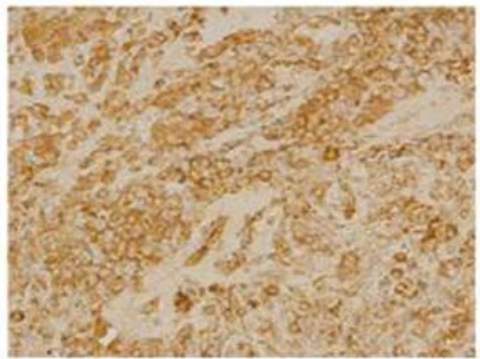

B

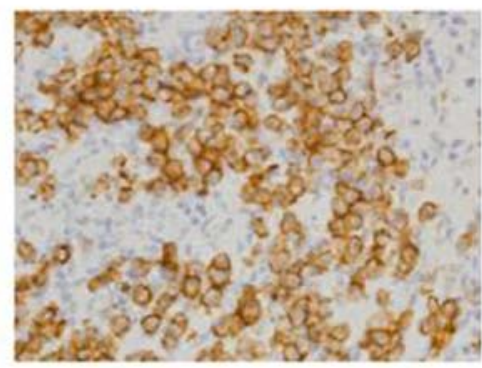

D

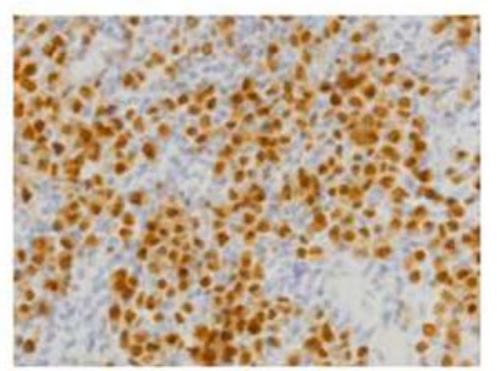

Figure 7. Representative histology of plasmocytoma cells of PDX tumor no. TGCT_002. (A) Tumor tissue slides stained with hematoxylin and eosin (magnification, x60). (B) Tumor tissue slides stained with plasma-cell marker CD138 antibody (magnification, x60). (C) Immunohistochemical staining of immunoglobulin 1 light chain in sections obtained from tumor tissue sample TGCT_002 (magnification, x40). (D) Tumor tissue slides stained with myeloma-associated oncogene MUM1/IRF-4 antibody (magnification, x60).

CD3, CD56 and CK20 (Fig. 7). The Ki-67 proliferation index was low $(<5 \%)$. The described morphological and phenotypic patterns allowed us to conclude that the analyzed tissue sample was infiltrated with tumor cells corresponding to the plasmocytoma of grade 1 expressing 1 light chain restriction.

\section{Discussion}

PDX models that realistically reflect patient heterogeneity and retain tumor biology represent a novel approach in the development of new treatment strategies. PDXs may also shed light on the pathways underlying the response of cancer cells to different 
treatment modalities, and enable the development of optimized combination therapies to overcome therapeutic resistance.

The aim of the present study was to establish and characterize TGCT patient-derived xenografts. These originated from retroperitoneal lymph node metastases infiltrated with TGCTs following previous cisplatin-based chemotherapy, in order to analyze novel treatment options for cisplatin-resistant testicular tumors. To the best of our knowledge, only a limited number of studies have investigated this field of TGCTs. The engraftment efficacy of primary xenotransplants in our patient cohort was $67 \%$. Several studies have analyzed factors associated with the successful engraftment of PDX tumors. It was demonstrated that PDX models derived from metastatic lesions have a higher engraftment rate, in comparison to PDXs derived from primary tumors $(24,25)$. In addition, a reduction of ischemic time and overall procedure time appearto be significant experimental parameters affecting successful implantation (12). Likewise, implantation in the same organ as the original tumor (orthotopic implantation), where the tumor develops in the same anatomical microenvironment, may also affect the success of PDX model establishment (26). Furthermore, using ECM as well as severe immunocompromised SCID mice has been demonstrated to improve successful engraftment in various cancer types (27-29). However, another study revealed that these factors, together with preoperative chemotherapy, did not have any significant influence on engraftment (12). The elapsed time for the successful engraftment in F1 animals was 40-55 days in the first case, and 25-40 days in the other case. In both cases, histological characteristics of the primary tumor were not retained, and lymphoma transformation was observed.

In clinical practice, the surgical resection of residual lymph nodal masses infiltrated with TGCTs after primary chemotherapy is usually performed. Histologically, these surgical specimens may contain mature teratoma in $\sim 30-40 \%$ of cases, fibrosis or necrosis in $48 \%$ of cases and cells in $10-20 \%$ of the patients (30-32). These findings are consistent with our results, where a surgical specimen obtained from the retroperitoneal lymph node metastasis of patient TGCT_002 was histologically characterized as teratocarcinoma. Notably, the study published by Brandli et al reported that the fibrous stromal cells adjacent to residual metastatic mature teratoma in lymph node specimens after chemotherapy have genetic abnormalities, similar to adjacent metastatic teratoma. These results indicated that both tumor components are derived from the same element of the original germ cell tumor or the same progenitor cell (33). Based on these data, we suggest that the transformation of the TGCTs PDX samples toward plasmocytoma/lymphoma observed in our study may be explained by the presence of totipotent EC as a common progenitor cell. The differentiation of seminoma cell line TCam2 towards EC-like cells was observed by Nettersheim et al, following the transplantation of TCam 2 cells into the seminiferous tubules of germ cell deficient (busulfan-treated) nude mice. The upregulation of SOX2 marker and downregulation of SOX17 expression was also reported (34). In addition, a similar transformation of seminoma cells towards embryonal carcinoma cells was observed in our laboratory following engraftment of TCam 2 cells into SCID mice.

Although it is generally known that NOD/SCID mice demonstrate a high incidence of thymic lymphomas with age (35), we excluded this possibility since qPCR analysis confirmed the presence of human $\beta$-globin-specific sequences in both successfully engrafted samples. However, Eppstein Barr virus (EBV)-related B-cell lymphomas have been observed in up to $68 \%$ of PDX tumor models derived from several types of cancer $(36,37)$. In the study by Chen et al, the engraftment of primary human hepatocellular carcinoma (HCC) tumor fragments or bulk tumor cell suspensions into immunodeficient mice resulted in lymphoid neoplasms, rather than HCC in 11 of the 21 established xenografts (38). Therefore, it was hypothesized that human solid tumor xenografts in immunodeficient mice are vulnerable to lymphomagenesis associated with TILs (tumor-infiltrating lymphocytes). TILs have been demonstrated likewise to be an important biomarker with prognostic and predictive features in different types of cancer. The increasing risk of relapse associated with low numbers of TILs has also been demonstrated in stage I seminomas (39). Cumulative data from murine studies has determined that myeloid lineage leukocytes, including tumor-associated macrophages, dendritic cells and myeloid-derived suppressor cells, are important factors in shaping the microenvironment via the factors they produce, towards either an immunostimulatory antitumor milieu or a wound healing tumor-promoting microenvironment (40). Therefore, a potential explanation for the lymphoma transformation observed in PDXs in our study may include TILs from the patient-originated tumors, which are transformed under new microenviroment conditions after their engraftment into immunodeficient recipient mice. It has been suggested that this potentially confounding process may be prevented by excluding leucocytes from the source tissue; for example, by treating mice with rituximab (a monoclonal antibody against B-cells) (41). However, there is no effective solution to prevent lymphoma transformation in PDXs at present (12).

In summary, we revealed that the PDX tumors in immunodeficient mice, derived from patients with lymph node metastases infiltrated with TGCTs (seminoma and teratocarcinoma) after previous cisplatin-based chemotherapy, transformed into lymphoma and subsequently into plasmocytoma. Lymphomagenesis represents new challenge in the establishment and serial propagation of PDX models derived from patients with testicular cancer.

\section{Acknowledgements}

We would like to acknowledge our collaborators Miroslava Matuskova, Roman Bohovic and Svetlana Miklikova from the Laboratory of Molecular Oncology, Cancer Research Institute, Biomedical Research Center of the Slovak Academy of Sciences, Bratislava, Slovakia for excellent technical assistance and animal maintenance.

\section{Funding}

The present study was supported by the Slovak Research and Development Agency [APVV-0016-11, APVV-15- 0086] and The Slovak Scientific Grant Agency [VEGA 1/0043/18].

\section{Availability of data and materials}

All data generated or analyzed during this study are included in this published article. 


\section{Authors' contributions}

KK, LK and MM designed the study, analyzed the data, and wrote the manuscript. KK, LK, SS and LT performed the research. ZK participated in the molecular analysis of tested samples. LK prepared Figs. 3 and 4. MC, PP, JM and MM provided clinical data. DP provided tissue samples. DM and LP performed the histological and immunohistochemical evaluations. LK and MM revised the manuscript and supervised the study. All authors discussed the results and reviewed the manuscript critically for important intellectual content. All the authors provided their final approval of the version to be published and agree to be accountable for all aspects of the research in ensuring that the accuracy or integrity of any part of the work are appropriately investigated and resolved.

\section{Ethics approval and consent to participate}

The Institutional Review Board of the National Cancer Institute, Bratislava, Slovakia (protocol IZLO1, chair: M. Mego) approved the present study and all patients provided written informed consent. Animal experiments were performed in the approved animal facility (license no. SK PC 14011) as approved by the Institutional Ethics Committee and by the national competence authority (State Veterinary and Food Administration of the Slovak Republic, registration no. Ro 3108/14-221) in compliance with the Directive 2010/63/EU of the European Parliament and the European Council and the Regulation 377/2012 on the protection of animals used for scientific purposes.

\section{Patient consent for publication}

Not applicable.

\section{Competing interests}

The authors declare that they have no competing interests.

\section{References}

1. Einhorn LH: Treatment of testicularcancer: A new and improved model. J Clin Oncol 8: 1777-1781, 1990.

2. Einhorn LH: General Motors Research Prizewinners Laureates Lectures. Charles F. Kettering Prize. Clinical trials in testicular cancer. Cancer 71: 3182-3184, 1993.

3. Motzer RJ, Amsterdam A, Prieto V, Sheinfeld J, Murty VV, Mazumdar M, Bosl GJ, Chaganti RS and Reuter VE: Teratoma with malignant transformation: Diverse malignant histologies arising in men with germ cell tumors. J Urol 159: 133-138, 1998.

4. Motzer RJ, Sheinfeld J, Mazumdar M, Bains M, Mariani T, Bacik J, Bajorin D and Bosl GJ: Paclitaxel, ifosfamide, and cisplatin second-line therapy for patients with relapsed testicular germ cell cancer. J Clin Oncol 18: 2413-2418, 2000.

5. Mardiak J, Salek T, Sycova-Mila Z, Obertova J, Hlavata Z, Mego M, Reckova M and Koza I: Paclitaxel plus ifosfamide and cisplatin in second-line treatment of germ cell tumors: A phase II study. Neoplasma 52: 497-501, 2005

6. De Giorgi U, Rosti G, Papiani G and Marangolo M: The status of high-dose chemotherapy with hematopoietic stem cell transplantation in germ cell tumor patients. Haematologica 87: 95-104, 2002.

7. Feldman DR, Patil S, Trinos MJ, Carousso M, Ginsberg MS Sheinfeld J, Bajorin DF, Bosl GJ and Motzer RJ: Progression-free and overall survival in patients with relapsed/refractory germ cell tumors treated with single-agent chemotherapy: Endpoints for clinical trial design. Cancer 118: 981-986, 2012.
8. Oechsle K, Kollmannsberger C, Honecker F, Mayer F, Waller CF, Hartmann JT, Boehlke I, Bokemeyer C and German Testicular Cancer Study Group: Long-term survival after treatment with gemcitabine and oxaliplatin with and without paclitaxel plus secondary surgery in patients with cisplatin- refractory and/or multiply relapsed germ cell tumors. Eur Urol 60: 850-855, 2011.

9. Mego M, Svetlovska D, Miskovska V, Obertova J, Palacka P, Rajec J, Sycova-Mila Z, Chovanec M, Rejlekova K, Zuzák P, et al: Phase II study of everolimus in refractory testicular germ cell tumors. Urol Oncol 34: 122.e17-e22, 2016

10. Daniel VC, Marchionni L, Hierman JS, Rhodes JT, Devereux WL, Rudin CM, Yung R, Parmigiani G, Dorsch M, Peacock CD and Watkins DN: A primary xenograft model of small-cell lung cancer reveals irreversible changes in gene expression imposed by culture in vitro. Cancer Res 69: 3364-3373, 2009.

11. Siolas D and Hannon GJ. Patient derived tumor xenografts: Transforming clinical samples into mouse models. Cancer Res 73: 5315-5319, 2013

12. Choi YY, Lee JE, Kim H, Sim MH, Kim KK, Lee G, Kim HI, An JY, Hyung WJ, Kim B, et al: Establishment and characterization of patient-derived xenografts as paraclinical models for gastric cancer. Sci Rep 6: 22172, 2016.

13. Fichtner I, Slisow W, Gill J, Becker M, Elbe B, Hilebrand T and Bibby M: Anticancer drug response and expression of molecular markers in early-passage xenotransplanted colon carcinomas. Eur J Cancer 40: 298-307, 2004.

14. Rubio-Viqueira B and Hidalgo M: Direct in vivo xenograft tumor model for predicting chemotherapeutic drug response in cancer patients. Clin Pharmacol Ther 85: 217-221, 2009.

15. DeRose YS, Wang G, Lin YC, Bernard PS, Buys SS, Ebbert MT, Factor R, Matsen C, Milash BA, Nelson E, et al: Tumor grafts derived from women with breast cancer authentically reflect tumor pathology, growth, metastasis and disease outcomes. Nat Med 17: 1514-1520, 2011.

16. Zhao X, Liu Z, Yu L, Zhang Y, Baxter P, Voicu H, Gurusiddappa S, Luan J, Su JM, Leung HC and Li XN: Global gene expression profiling confirms the molecular fidelity of primary tumor-based orthotopic xenograft mouse models of medulloblastoma. Neuro Oncol 14: 574-583, 2012.

17. Houghton JA, Houghton PJ and Green AA: Chemotherapy of childhood rhabdomyosarcomas growing as xenografts in immune-deprived mice. Cancer Res 42: 535-539, 1982.

18. Talmadge JE, Singh RK, Fidler IJ and Raz A: Murine models to evaluate novel and conventional therapeutic strategies for cancer. Am J Pathol 170: 793-804, 2007.

19. Abraham D, Abri S, Hofmann M, Höltl W and Aharinejad S: Low dose carboplatin combined with angiostatic agents prevents metastasis in human testicular germ cell tumor xenografts. J Urol 170: 1388-1393, 2003.

20. Castillo-Avila W, Piulats JM, Garcia Del Muro X, Vidal A, Condom E, Casanovas O, Mora J, Germà JR, Capellà G, Villanueva A and Viñals F: Sunitinib inhibits tumor growth and synergizes with cisplatin in orthotopic models of cisplatin-sensitive and cisplatin-resistant human testicular germ cell tumors. Clin Cancer Res 15: 3384-3395, 2009.

21. de Vries G, Rosas-Plaza X, van Vugt MA, Suurmeijer A, Gietema JA and de Jong S: Development of testicular cancer patient derived xenograft models to test combination therapies targeting PI3K/Akt and MDM2. Cancer Res 77 (Suppl 13): 3861, 2017.

22. Livak KJ and Schmittgen TD: Analysis of relative gene expression data using real-time quantitative PCR and the 2(-Delta Delta C(T)) method. Methods 25: 402-408, 2001.

23. Matuskova M, Kozovska Z, Toro L, Durinikova E, Tyciakova S, Cierna Z, Bohovic R and Kucerova L: Combined enzyme/prodrug treatment by genetically engineered AT-MSC exerts synergy and inhibits growth of MDA-MB-231 induced lung metastases. J Exp Clin Cancer Res 34: 33, 2015.

24. Némati F, Sastre-Garau X, Laurent C, Couturier J, Mariani P, Desjardins L, Piperno-Neumann S, Lantz O, Asselain B, Plancher C, et al: Establishment and characterization of a panel of human uveal melanoma xenografts derived from primary and/or metastatic tumors. Clin Cancer Res 16: 2352-2362, 2010.

25. Sivanand S, Peña-Llopis $S$, Zhao $H$, Kucejova B, Spence $P$, Pavia-Jimenez A, Yamasaki T, McBride DJ, Gilen J, Wolff NC, et al: A validated tumor graft model reveals activity of dovitinib against renal cell carcinoma. Sci Transl Med 4: 137ra75, 2012. 
26. Wang $\mathrm{X}, \mathrm{Fu} \mathrm{X}$ and Hoffman RM: A new patient-like metastatic model of human lung cancer constructed orthotopically with intact tissue via thoracotomy in immunodeficient mice. Int J Cancer 51: 992-995, 1992.

27. Shuhendler AJ, Prasad P, Cai P, Hui KK, Henderson JT, Rauth AM and Wu XY: Matrigel alters the pathophysiology of orthotopic human breast adenocarcinoma xenografts with implications for nanomedicine evaluation. Nanomedicine 9: 795-805, 2013

28. Read M, Liu D, Duong CP, Cullinane C, Murray WK Fennell CM, Shortt J, Westerman D, Burton P, Clemons NJ and Phillips WA: Intramuscular transplantation improves engraftment rates for esophageal patient-derived tumor xenografts. Ann Surg Oncol 23: 305-311, 2016.

29. Fujii E, Suzuki M, Matsubara K, Watanabe M, Chen YJ, Adachi K, Ohnishi Y, Tanigawa M, Tsuchiya M and Tamaoki N Establishment and characterization of in vivo human tumor models in the NOD/SCID/gamma(c)(null) mouse. Pathol Int 58: 559-567, 2008

30. Krege S, Beyer J, Souchon R, Albers P, Albrecht W, Algaba F, Bamberg M, Bodrogi I, Bokemeyer C, Cavallin-Ståhl E, et al: European consensus conference on diagnosis and treatment of germ cell cancer: A report of these condmeeting of the european germ cell cancer consensus group (EGCCCG): Part I. Eur Urol 53: 478-496, 2008.

31. Beck SD and Foster RS: Long-term outcome of retroperitoneal lymph node dissection in the management of testis cancer. World J Urol 24: 267-272, 2006.

32. Oldenburg J, Alfsen GC, Lien HH, Aas N, Waehre H and Fossa SD: Postchemotherapy retroperitoneal surgery remains necessary in patients with nonseminomatous testicular cancer and minimal residual tumor masses. J Clin Oncol 21: 3310-3317, 2003.

33. Brandli DW, Ulbright TM, Foster RS, Cummings OW, Zhang S, Sweeney CJ, Eble JN and Cheng L: Stroma adjacent to metastatic mature teratoma after chemotherapy for testicular germ cell tumors is derived from the same progenitor cells as the teratoma. Cancer Res 63: 6063-6068, 2003.
34. Nettersheim D, Westernstroer B, Gillis AJM, Looijenga LHJ, Schlatt $\mathrm{S}$ and Schorle $\mathrm{H}$ : Chanages in the microenviroment affect differentiation of the seminoma cell line TCam-2 and infusion into the mouse testis provides a model for the study CIS 7th Copenhagen Workshop on CIS-Testis and Germ Cell Cancer Abstracts: abs. 24, 2010

35. Prochazka M, Gaskins HR, Shultz LD and Leiter EH: The nonobese diabetic scid mouse: Model for spontaneous thymomagenesis associated with immunodeficiency. Proc Natl Acad Sci USA 89: 3290-3294, 1992.

36. Zhang L, Liu Y, Wang X, Tang Z, Li S, Hu Y, Zong X, Wu X, $\mathrm{Bu} \mathrm{Z}, \mathrm{Wu} \mathrm{A}$, et al: The extent of inflammatory infiltration in primary cancer tissues is associated with lymphomagenesis in immunodeficient mice. Sci Rep 5: 9447, 2015.

37. John T, Yanagawa N, Kohler D, Craddock KJ, BandarchiChamkhaleh B, Pintilie M, Sykes J, To C, Li M, Panchal D, et al: Characterization of lymphomas developing in immunodeficient mice implanted with primary human non-small cell lung cancer. J Thorac Oncol 7: 1101-1108, 2012.

38. Chen K, Ahmed S, Adeyi O, Dick JE and Ghanekar A Humansolid tumor xenografts in immunodeficient mice are vulnerable to lymphomagenesis associated with epstein-barr virus. PLoS One 7: e39294, 2012.

39. Parker C, Milosevic M, Panzarella T, Banerjee D, Jewett M, Catton C, Tew-George B, Gospodarowicz M and Warde P: The prognostic significance of the tumour infiltrating lymphocyte count in stage I testicular seminoma managed by surveillance. Eur J Cancer 38: 2014-2019, 2002.

40. Salgado R, Denkert C, Demaria S, Sirtaine N, Klauschen F, Pruneri G, Wienert S, Van den Eynden G, Baehner FL, PenaultLlorca F, et al: The evaluation of tumor-infiltrating lymphocytes (TILs) in breast cancer: Recommendations by an international TILs working group 2014. Ann Oncol 26: 259-271, 2015.

41. Fujii E, Kato A, Chen YJ, Matsubara K, Ohnishi Y and Suzuki M: Characterization of EBV-related lymphoproliferative lesions arising in donor lymphocytes of transplanted human tumor tissues in the NOG mouse. Exp Anim 63: 289-296, 2014. 